\title{
Evaluación del Control Interno a la Gestión de Inventarios de IMPORELLANA S.A en Santo Domingo, periodo 2017
}

\section{Internal Control Evaluation to the Inventory Managment of Imporellana S.A in Santo Domingo, period 2017}

\author{
*Ximena Leticia García Zambrano1 , **Jhoanna Lisbeth Sánchez Enríquez ${ }^{1}$ \\ ${ }^{1}$ Pontificia Universidad Católica del Ecuador Sede Santo Domingo \\ *gzxl@pucesd.edu.ec;**jlsancheze@pucesd.edu.ec
}

Fecha de recepción: 14/11/2018

Fecha de aceptación: 25/02/2019

Publicado: $30 / 06 / 2019$

\section{Resumen}

El presente artículo tiene como fin evaluar el control interno a la gestión de inventarios de “IMPORELLANA S.A" en el periodo 2017. Para lograr este objetivo se utilizó un enfoque cuantitativo y cualitativo, con una investigación de tipo descriptivo-explicativo. La población estuvo conformada por todo el personal de la empresa, así como los proveedores y clientes del último semestre del periodo 2017. Para conocer la situación actual de la empresa se realizó el levantamiento de información a través de técnicas de recogida de información como encuestas y entrevistas acerca de la administración y control de los inventarios que maneja la entidad. Luego se realizó la evaluación del control interno en donde se aplicó el cuestionario COSOERM que permitió determinar el nivel de confianza y riesgo de la gestión de inventarios. En la identificación de riesgos con base en pruebas de cumplimiento, se determinó el nivel de eficiencia y eficacia de los procesos de compras, recepción, almacenamiento y ventas. En el informe de auditoría se detallaron los hallazgos encontrados que afectan al control interno, con su respectiva conclusión y recomendación. Los resultados obtenidos demuestran que el problema del control de inventarios de la empresa se centra en la deficiente planificación de las compras y mecanismos de control del movimiento de la mercadería.

Palabras clave: Control interno, Inventarios, Compras, Recepción, Almacenamiento, Ventas. 


\begin{abstract}
This article has as purpose evaluate the Internal Control to the Inventory Managment of Imporellana S.A in Santo Domingo, period 2017. To achieve this goal was used a quantitative and qualitative approach with a descriptive-explanatory research. The population was formed by the whole staff of the company, as well as suppliers and customers of the last half of the 2017 period. To know the current situation of the company it was realized the information gatherin through data collection techniques such as surveys and interviews about the administration and inventory control that handles the entity.Then it was done the evaluation of the internal control where te COSO-ERM questionnaire was applied to establish the level of trust and risk of inventory management. In the identification of risks base on compliance tests, it was determined the level of efficiency and effectiveness of the purchases, reception, storage and sales processes. In the audit report were detailed the findings found that affect the internal control, with its respective conclusion and recommendation. The results obtained show that the problem of the company inventory control it focuses on the poor planning of purchases and mechanisms of control of the movement of the merchandise.
\end{abstract}

Keywords: Internal Control, Inventory, Purchases, Reception, Storage, Sales.

\title{
Introducción
}

A lo largo del tiempo, debido a la evolución de la tecnología, los procesos de las organizaciones se hicieron cada vez más complejos, provocando nuevos problemas como fraudes o fallos en los sistemas informáticos, siendo necesario establecer sistemas que controlen y mejoren los procesos operacionales. Por este motivo, el control interno surgió como una necesidad para apoyar el logro de los objetivos organizacionales y como un medio indispensable para llevar a cabo un control eficaz (Vásquez, 2016).

Se puede decir entonces que el control interno es un conjunto de procedimientos que debe ser llevado a cabo por todos los miembros de la organización, con el objetivo de proporcionar 
un grado de seguridad razonable en cuanto a la eficiencia de las operaciones, confiabilidad de la información financiera y cumplimiento de las leyes aplicables; disminuyendo de esta manera los posibles riesgos que pueden afectar a la entidad (Paiva, 2013).

A pesar que no todas las empresas tienen un sistema de control interno, su implementación ha aumentado en los últimos años, ya que representa una parte importante dentro de toda entidad porque permite medir y aumentar la eficiencia y productividad operativa así como la confiabilidad de la información contable, teniendo en cuenta que esto se logrará con la ayuda de cada miembro que conforma la estructura organizacional, ayudando de esta manera a una adecuada toma de decisiones (Paiva, 2013).

En estudios recientes, demuestran que aunque una entidad objeto de estudio práctico presente un control interno que se evalúe como alto, se pueden detectar debilidades importantes en su gestión. Ya que a pesar de que el control se encuentre establecido en la mayoría de las actividades, existe la necesidad de incrementar este en todas las áreas de la empresa, ya que de lo contrario, se incurrirían en altos gastos presupuestarios por concepto de tiempo, fuerza de trabajo y posibles pérdidas económicas (Vega, Lao y Nieves, 2017).

Los procesos de control interno se deben implementar en todas las empresas, sean estas grandes o pequeñas, con o sin fines de lucro. Por ende, toda empresa que se dedique a la compraventa de mercadería debe tener un buen manejo y control interno de sus inventarios, ya que la deficiencia en el control interno del área de compras y del área contable afectará las ventas y por ende la rentabilidad de la empresa. Además aquellas empresas que no efectúen procesos de control interno ven mellada su productividad en un $25 \%$ (Obispo y Gonzales, 2015).

Teniendo en cuenta que el inventario es el activo más significativo de las empresas comerciales, nace la importancia de hacer una evaluación del sistema de control interno a la gestión de inventarios, con el propósito de mejorar la administración y control de la 
mercadería dentro de la entidad. Por esta razón, surge la necesidad de reformar los procesos y políticas de compra, recepción, almacenamiento, venta de la mercadería, que contribuirá a la toma de decisiones gerenciales a favor de la empresa y de sus clientes, mejorando de esta manera la eficiencia en las operaciones de la organización.

En el Ecuador, el sector comercial tiene una gran importancia, ya que el crecimiento económico está relacionado con el incremento de las actividades comerciales. En el año 2016, las empresas comerciales ocuparon el segundo puesto como el sector empresarial que más empleo genera, con el $26,67 \%$ de personas empleadas del total investigado (Instituto Nacional de Estadística y Censos [INEC], 2016).Sin embargo, en el país, la mayoría de las empresas comerciales administran y controlan sus inventarios de manera empírica, ya sea por desconocimiento o falta de control en las diferentes actividades de la gestión de inventarios; lo que provoca clientes insatisfechos, disminución de las ventas y problemas financieros.

Santo Domingo, debido a su ubicación geográfica, es una provincia con una gran actividad comercial, dentro de la cual, debido a la evolución y demanda de la tecnología, se destacan los locales comerciales dedicados a la venta de electrodomésticos. En la actualidad, el sector comercial se dedica a la importación de equipos que debido a la innovación tecnológica poseen un tiempo corto de vida útil, lo que provoca una demanda constante por parte de los consumidores. (El Telégrafo, 2012). En los últimos años, debido a la exigencia del mercado en cuanto a la actualización de la tecnología, las empresas se esfuerzan cada vez más por diversificar la gama de productos para así satisfacer las necesidades de sus clientes.

Sin embargo, en contraposición, las empresas deben soportar el posible riesgo de exceso o sobreabastecimiento de stock, ya sea por cambios en los patrones de compra de los consumidores o por una mala planificación en el proceso de compras; generando que los activos corrientes de las empresas estén comprometidos con el inventario, lo cual a su vez compromete la liquidez de las empresas. 
Un deficiente control interno del inventario conlleva a grandes consecuencias en empresas donde la mercadería representa la principal fuente de ingresos, ya que la rotación de los misma se convierte en dinero en efectivo de manera inmediata una vez que se ha efectuado la venta. Algunas empresas, a pesar de tener una buena rotación de inventarios, presentan artículos permanentes en bodega, los cuales pueden volverse obsoletos, generando pérdidas significativas en las utilidades de la empresa (Quinde y Ramos, 2018).

IMPORELLANA S.A es una de las empresas que se dedica a la importación y comercialización de electrodomésticos en la ciudad de Santo Domingo. La empresa fue fundada en el año 1995 por el Sr. Robert Orellana, en un pequeño local ubicado en las calles 3 de julio y Ambato. Posteriormente, en el año 2011, debido al incremento de la demanda se trasladó a un local más amplio luego de constituirse como sociedad anónima.

Debido al crecimiento de la empresa y a la aplicación de métodos empíricos en los procesos, se presentan varios problemas referentes a la gestión de inventarios, de los cuales se destacan los siguientes: Falta de planificación al momento de realizar los pedidos, lo que luego genera roturas de stock. Desorden en la recepción de mercadería, referente a la cantidad y calidad de la misma. Organización deficiente de la mercadería tanto en el almacén como en bodega, lo que dificulta encontrar la mercadería. Desorden en el registro de la salida de la mercadería lo que provoca un desconocimiento del saldo real del inventario.

La presente investigación es de gran utilidad para la empresa ya que al mejorar el control interno de los inventarios, el propietario de la organización podrá tomar decisiones acertadas que ayudarán a disminuir los costos de adquisición y almacenamiento de la mercadería, así como evitar sobreabastecimientos o escasez de los productos y por ende brindar un servicio de calidad a los clientes.

IMPORELLANA actualmente, no tiene implementado procesos específicos para la gestión de inventarios, sin embargo sí posee ciertas directrices como políticas y delegación de 
funciones que aportan a la consecución de los objetivos organizacionales. Por consiguiente, es necesario establecer un modelo de control interno administrativo y contable que asegure la eficiencia de las operaciones de la empresa, así como la fiabilidad de la información financiera.

Por lo tanto, el objetivo principal de esta investigación es evaluar el sistema de control interno de la gestión de inventarios de la empresa IMPORELLANA, a través del levantamiento de información de los procesos actuales, la evaluación del control interno con base en el COSO ERM, la identificación de riesgos, para luego plasmar en un informe los hallazgos encontrados en el trabajo de auditoría.

\section{Materiales y métodos}

El enfoque cuantitativo usa la recopilación de datos para probar hipótesis con base en la medición numérica y el análisis estadístico. Por otro lado el enfoque cualitativo emplea la recolección de datos sin medición numérica para revelar o mejorar preguntas de investigación en el proceso de interpretación (Hernández, Fernández y Baptista, 2014).

Por tal motivo, la presente investigación tuvo un enfoque mixto, ya que se utilizó el enfoque cuantitativo en la tabulación de las encuestas dirigidas al personal, proveedores y clientes de IMPORELLANA S.A. Y un enfoque cualitativo cuando se realizaron las entrevistas al gerente y al contador externo de la empresa con el objetivo de obtener información relacionada con el área de inventarios.

Se utilizó un diseño de investigación no experimental porque se observaron los procesos que involucra la gestión de inventarios tal y como suceden dentro de la empresa IMPORELLANA S.A, para posteriormente analizar la eficiencia de los mismos. La investigación experimental se define como el estudio que se realiza sin manipular deliberadamente las variables para ver su efecto sobre otras variables. Es decir, se observa el 
fenómeno tal como se da en su contexto natural para posteriormente analizarlo, sin variar de forma intencional las variables (Hernández et al., 2014).

En el presente trabajo se utilizaron las siguientes tipos de investigación.

"La investigación descriptiva consiste en describir una situación real natural mediante la observación sistemática no participante o valiéndose de preguntas a una muestra de personas capaces de proporcionar la información deseada sobre opiniones, comportamientos o circunstancias" (Belmonte y González, 2013, p.103). Es decir, su objetivo es únicamente recopilar información sobre las variables estudiadas más no establecer como éstas se relacionan entre sí.

La investigación explicativa está dirigida a responder por las causas de los eventos y fenómenos físicos o sociales. Se enfoca en explicar porque un fenómeno y en qué condiciones se manifiesta, o porqué se relacionan dos o más variables (Hernández et al., 2014, p.85). Su principal interés es explicar los motivos por los cuales ocurre un fenómeno o mediante qué circunstancias se presenta.

La investigación fue descriptiva-explicativa, ya que se detallaron cada uno de los procedimientos y tareas de los individuos involucrados en la compra, almacenamiento y salida de la mercadería, con el fin de posteriormente examinar el Sistema de Control Interno y establecer relaciones causa-efecto de su aplicación en la empresa.

Según Deza y Muñoz (2012) "la población es la totalidad del fenómeno a estudiar, la misma que posee una característica en común" (p. 61). La población para el caso de estudio fue de 236, constituida por el gerente de la empresa, el contador externo, 11 empleados, 27 proveedores y 126 clientes externos. Debido a que la población es infinita se aplicó la fórmula del cálculo de la muestra dando como resultado 146 personas que fueron distribuidas de la siguiente forma: el gerente, el contador externo, once empleados, veintisiete proveedores y ciento seis clientes, correspondientes al último trimestre del periodo 2017. 
Las técnicas utilizadas para recopilar la información fueron la observación no experimental de los procesos referentes a la gestión de inventarios, entre ellos el proceso de compras, recepción, almacenamiento, salida y registro de la mercadería. La entrevista estructurada al gerente de la empresa "IMPORELLANA S.A", para obtener información oportuna referente a la estructura organizacional, las políticas y el procedimiento que llevan a cabo para realizar las compras y almacenamiento de la mercadería; con el propósito de efectuar un análisis del problema desde un punto de vista gerencial. Y la encuesta asistida con preguntas cerradas a los empleados, proveedores y clientes de la empresa relacionados con la gestión de inventarios.

\section{Resultados}

Fase I: Levantamiento de información del proceso de la gestión de inventarios de IMPORELLANA S.A.

Con el fin de obtener información adecuada acerca de la estructura organizacional, las políticas y las actividades realizadas al momento de efectuar las compras, de recibir o almacenar la mercadería en las bodegas y de registrar las entradas y salidas de stock; se realizaron dos entrevistas, una al gerente de la empresa y otra al contador externo; y tres encuestas a los empleados, proveedores y clientes.

\section{Análisis de la entrevista dirigida al gerente}

Como resultado de la entrevista realizada al Gerente se pudo evidenciar que la empresa no posee un organigrama que refleje los niveles jerárquicos existentes dentro de la entidad. Además, a pesar de que cada empleado tiene un cargo, la empresa no cuenta con un manual de funciones que especifique las tareas y actividades que cada trabajador debe realizar. Se puede comprobar también que la mayoría del personal que labora en la empresa fue contratado por motivos de afinidad, observando en el rol de pagos proporcionado por la 
empresa que casi todos los empleados son familiares del propietario; por lo cual se concluye que el personal no fue contratado por sus habilidades sino por vínculo familiar.

De igual forma, la empresa no posee un manual de políticas y procedimientos para la gestión de inventarios que guie a los empleados en las actividades que deben realizar de forma sistemática para la compra, almacenamiento, venta y registro de la mercadería. Una falencia importante que se pudo detectar, es que la empresa no planifica sus compras ya que realizan la adquisición de los productos cuando se dan cuenta que el stock de los mismos está en cero en el sistema, lo que luego ocasiona que pierdan ventas ya que no satisfacen las necesidades de sus clientes debido al desabastecimientos de ciertos productos.

\section{Análisis de la entrevista dirigida al contador externo}

Con la entrevista realizada al contador externo IMPORELLANA S.A se logró comprobar que los inventarios de la empresa sí se registran de acuerdo a las normativas contables vigentes, y que además utilizan el sistema de inventario permanente para registrar la adquisición y ventas de la mercadería. Sin embargo, el contador comentó que la empresa no utiliza actualmente ningún método de valoración de inventarios para controlar las unidades de los productos en el almacén.

También, el contador considera que el sistema contable "Anfibius" utilizado por la empresa no proporciona información confiable para los registros de las transacciones de mercadería, ya que han existido ocasiones en que la persona encargada de registrar las compras y ventas dentro de la empresa, ingresa de manera incorrecta estos movimientos; dando como resultado un saldo poco razonable de la cuenta inventarios.

\section{Análisis de la encuesta dirigida a los proveedores}

Como resultado de la encuesta realizada a los proveedores de IMPORELLANA S.A se pudo comprobar que los empleados sí verifican la calidad y cantidad de la mercadería que llega a la 
empresa, a través de los documentos correspondientes que se realizaron previamente como la guía de remisión que detalla el número de unidades solicitadas.

Sin embargo, cuando un pedido supera la cantidad de productos solicitados, se devuelve todo el pedido de la mercadería, ocasionada que la empresa quede desabastecida de ese producto por un periodo de tiempo hasta que vuelvan a recibir el pedido correcto o mientras buscan otro proveedor.

\section{Análisis de la encuesta dirigida a los empleados}

Con la encuesta realizada a los empleados de IMPORELLANA S.A se pudo conocer que a pesar de que la empresa da a conocer a los empleados las actividades que cada uno ellos deben realizar, existen ocasiones en que los mismos deben ejecutar funciones que no corresponden a su cargo debido a una mala asignación de horarios para la hora de almuerzo por parte de la gerencia, lo que ocasiona que la secretaria realice tareas que no pertenecen a su puesto de trabajo.

Se pudo comprobar también, que la empresa no lleva un registro de la mercadería que ingresa y sale de bodega, debido a que no existe una persona específica con el puesto de bodeguero, sino que la mayoría de vendedores realizan las funciones que esta persona realizaría. De igual forma, la empresa realiza tomas físicas de inventarios solo una vez al año, lo que ocasiona que existan constantemente diferencias entre el saldo de bodega y el saldo que entrega el sistema contable.

Además, se consiguió evidenciar que el gerente no comunica a los empleados los objetivos o metas organizacionales que se desean alcanzar. Del mismo modo, gerencia no realiza una evaluación o monitoreo del desempeño de sus colaboradores, que le permita conocer el nivel de eficiencia y eficacia con la que sus empleados realizan su trabajo.

\section{Análisis de la encuesta dirigida a los clientes}


Como resultado de la encuesta realizada a los clientes de IMPORELLANA S.A se pudo comprobar que en ocasiones los clientes de la empresa no encuentran el producto que buscan debido a la mala planificación de las compras por parte de la empresa, llegando a ofrecer en ocasiones productos que estaban en el sistema pero no en bodega, lo que provoca insatisfacción en los clientes y por ende una disminución de las ventas.

Sin embargo, la mayoría de los clientes reconocen que los empleados de la organización sí poseen los conocimientos necesarios para su puesto de trabajo, porque cada vez que acuden a la empresa son atendidos de una buena manera, ya que el personal tiene conocimiento de las características, usos y beneficios para el cliente de los productos que ofrece la empresa; a más de conocer las formas de pago a las que pueden acceder los clientes.

Además, se logró determinar que el despacho de la mercadería a los clientes es en su mayoría calificado como bueno por parte de los mismos, debido a que los empleados sí verifican la mercadería antes de que esta sea entregada, también porque no existen demoras en la entrega de los productos ni tampoco existe un número considerable de devoluciones de mercadería por parte de los clientes.

\section{Análisis del proceso de compras}

El proceso inicia con la visita de los proveedores a las instalaciones de la empresa. La secretaria revisa en el sistema contable de la empresa la cantidad de la mercadería existente. Los proveedores comunican si existen promociones especiales de ciertos artículos. La empresa decide si tomar o no la oportunidad de promoción.

En caso de ser una compra de carácter urgente, el gerente se comunica con los proveedores de la mercadería requerida vía línea telefónica, para realizar el pedido. Una vez acordada la cantidad, el precio, los descuentos, y el plazo de pago de la mercadería; el proveedor elabora la nota de pedido. 
Finalmente, para el pago de la mercadería algunos proveedores tienen como política el pago anticipado de la mercadería que van a enviar (prepago), para lo cual la empresa efectúa el pago en el momento en que se realiza el pedido. La otra modalidad de pago es a crédito la cual puede ser a 3, 6 u 8 meses, según la política de plazo de pago del proveedor.

\section{Análisis del proceso de almacenamiento y recepción}

El proceso inicia con el arribo de la mercadería a las instalaciones de la empresa, la cual posee dos bodegas ubicadas contiguamente al almacén en donde el asistente de bodega recibe los productos con la copia de la nota de pedido y la factura verificando que la misma se encuentre en buen estado y además que la cantidad sea la que previamente fue solicitada.

Si el pedido es el correcto, el asistente de bodega firma la guía de remisión, luego los empleados almacenan la mercadería y la ordenan en el lugar correspondiente en la bodega, para luego autorizar a gerencia la firma de la factura. Por el contrario si llega a existir alguna anomalía en el pedido recibido los empleados informan el problema al gerente para que se comunique con los proveedores para llegar a un acuerdo. Luego con la copia de la factura de la compra la secretaria procede a registrar y codificar la nueva mercadería en el sistema contable de la empresa.

\section{Análisis del proceso de ventas}

El proceso inicia con la visita de los clientes a las instalaciones de la empresa. Los vendedores reciben al cliente y le preguntan qué producto desea comprar. El cliente da las especificaciones del producto que quiere adquirir. El empleado ofrece diferentes marcas y modelos del producto que solicitó, posteriormente explica de forma detallada las características del artículo.

Luego la secretaria realiza la cotización de los productos que el cliente está interesado en comprar, y junto con el gerente se llega a un acuerdo en el precio de los artículos cotizados. Si 
el cliente aún está interesado en realizar la compra, la secretaria le informa las formas de pago que ofrece la empresa. Si la venta es al contado, la secretaria genera la factura y realiza el cobro de la mercadería vendida. Si la venta es a crédito se realiza la recepción de los documentos necesarios para otorgar el crédito, se establece el plazo de pago, se genera la factura y una copia que es entregada al cliente.

El vendedor que atendió al cliente empaca el producto que se va a vender y verifica que el mismo funcione y este completo, para posteriormente entregar el producto al comprador. Finalmente, la secretaria registra en el sistema contable de la empresa la salida de los productos en el módulo denominado "inventarios". Cabe recalcar que la empresa no registra la salida de la mercadería de bodega, ya que no llevan ningún documento o reporte que les permita controlar los movimientos internos que realizan.

Fase II: Identificación de riesgos a través de los instrumentos de evaluación del sistema de control interno.

Para evaluar el sistema de control interno se aplicó el cuestionario COSO ERM que permitió determinar el nivel de confianza y riesgo de la gestión de inventarios en cada uno de los ocho componentes que lo conforman.

Tabla 1.

Matriz de calificación de riesgos y nivel de confianza por componentes

\begin{tabular}{lcccccc}
\hline \multicolumn{1}{c}{ Componentes } & $\begin{array}{c}\text { Respuestas } \\
\text { Positivas }\end{array}$ & $\begin{array}{c}\text { Respuestas } \\
\text { Negativas }\end{array}$ & $\begin{array}{c}\text { Total } \\
\text { Preguntas }\end{array}$ & $\begin{array}{c}\text { Porcentaje } \\
\text { Confianza }\end{array}$ & $\begin{array}{c}\text { Porcentaje de } \\
\text { Riesgo }\end{array}$ & Confiabilidad \\
\hline $\begin{array}{l}\text { Ambiente de } \\
\text { control }\end{array}$ & 1 & 4 & 5 & $20 \%$ & $80 \%$ & No confiable \\
\hline $\begin{array}{l}\text { Establecimiento de } \\
\text { objetivos }\end{array}$ & 1 & 4 & 5 & $20 \%$ & $80 \%$ & No confiable \\
\hline $\begin{array}{l}\text { Identificación de } \\
\text { eventos }\end{array}$ & 4 & 4 & 8 & $50 \%$ & $50 \%$ & Aceptable \\
\hline $\begin{array}{l}\text { Valoración de } \\
\text { riesgos }\end{array}$ & 3 & 2 & 5 & $60 \%$ & $40 \%$ & Aceptable \\
\hline Respuesta al riesgo & 3 & 2 & 5 & $60 \%$ & $40 \%$ & Aceptable \\
\hline $\begin{array}{l}\text { Actividades de } \\
\text { control }\end{array}$ & 3 & 3 & 6 & $50 \%$ & $50 \%$ & Aceptable \\
\hline $\begin{array}{l}\text { Información y } \\
\text { comunicación }\end{array}$ & 3 & 3 & 6 & $50 \%$ & $50 \%$ & Aceptable \\
\hline $\begin{array}{l}\text { Monitoreo y } \\
\text { evaluación }\end{array}$ & 1 & 4 & 5 & $20 \%$ & $80 \%$ & No confiable \\
\hline TOTAL & $\mathbf{1 9}$ & $\mathbf{2 6}$ & $\mathbf{4 5}$ & & & \\
\hline
\end{tabular}

Elaborado por: García, X. y Sánchez, J. 


$$
\begin{gathered}
\text { Nivel de confianza }=\frac{\text { Respuestas positivas }}{\text { Total respuestas }}=\frac{19}{45}=42,22 \% \\
\text { Nivel de riesgo }=\frac{\text { Respuestas negativas }}{\text { Total respuestas }}=\frac{26}{45}=57,78 \%
\end{gathered}
$$

De acuerdo con el nivel de confianza obtenido en el Cuestionario de Control Interno COSO ERM de 42,22\%, la empresa se encuentra en el nivel 3 de riesgo, con el cual se procedió a calcular la muestra que dio como resultado 60. Por lo tanto, serán sujetos a revisión 60 comprobantes relacionados con el ciclo de inventarios, seleccionando documentos con los valores más altos.

Tabla 2.

Nivel de confianza y riesgo general

\begin{tabular}{cccc}
\hline Rangos & Nivel de confianza & Calificación & Nivel de riesgo \\
\hline $75 \%-95 \%$ & Alto & & 1 \\
\hline $50 \%-74 \%$ & Moderado & & 2 \\
\hline $15 \%-49 \%$ & Bajo & $42,22 \%$ & 3 \\
\hline
\end{tabular}

Elaborado por: García, X. y Sánchez, J.

$$
\text { Muestra }=\frac{\text { nivel de riesgo }}{\text { Grado de error }}=\frac{3}{0,05}=60
$$

Fase III: Ejecución de procedimientos para evaluar el sistema de control interno en la gestión de inventarios.

Para evaluar el control interno de la empresa se procedió a examinar 60 partidas claves de la gestión de inventarios como son las compras y ventas, así como los procesos de recepción y almacenamiento, con el objetivo de identificar el nivel de cumplimiento de las políticas en cada uno de las actividades.

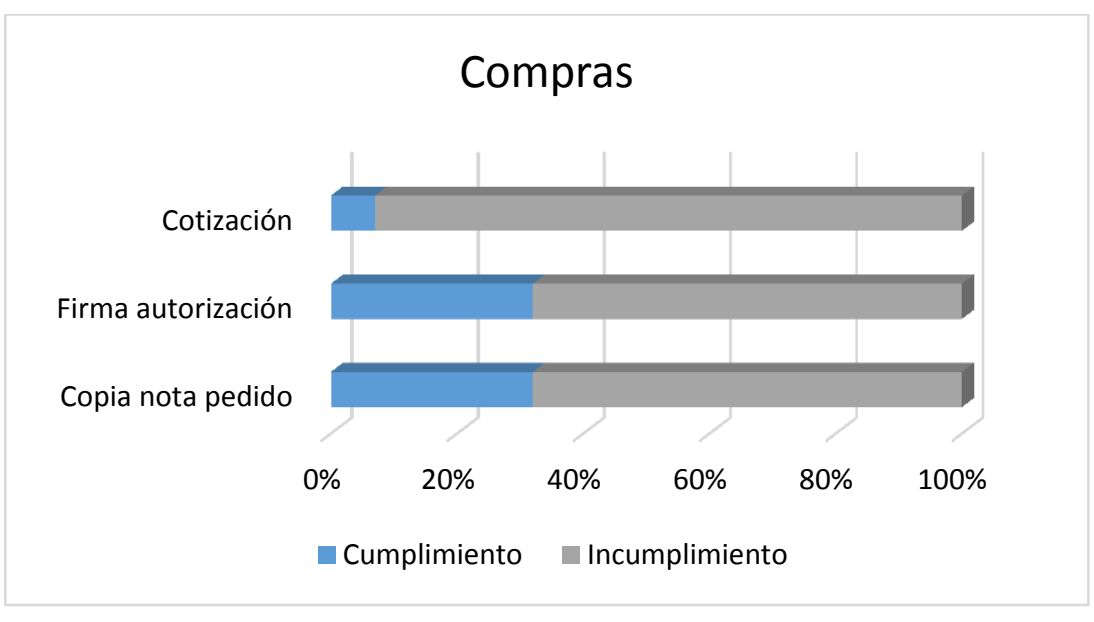

Figura 1. Cumplimiento de políticas en el proceso de compras. Investigación de campo. 


\section{Nivel de cumplimiento de políticas en el proceso de compras.}

El 68\% de las facturas de compras revisadas no cumplen con la emisión de la copia de la nota de pedido, por ende la política de firma de autorización tiene el mismo porcentaje de incumplimiento. Según las políticas señaladas en el proceso de compras de IMPORELLANA S.A, la documentación requerida es la copia de la nota de pedido que efectúan los proveedores, para tener un respaldo de los solicitado en el momento de la recepción de la mercadería. Sin embargo, la mayoría de las empresas realizan las notas de pedido de manera electrónica, por lo que IMPORELLANA S.A no puede solicitar una copia de la nota de pedido que realizan los proveedores. Lo que provoca incertidumbre al momento de la recepción de la mercadería al no tener un respaldo del pedido que se realizó previamente.

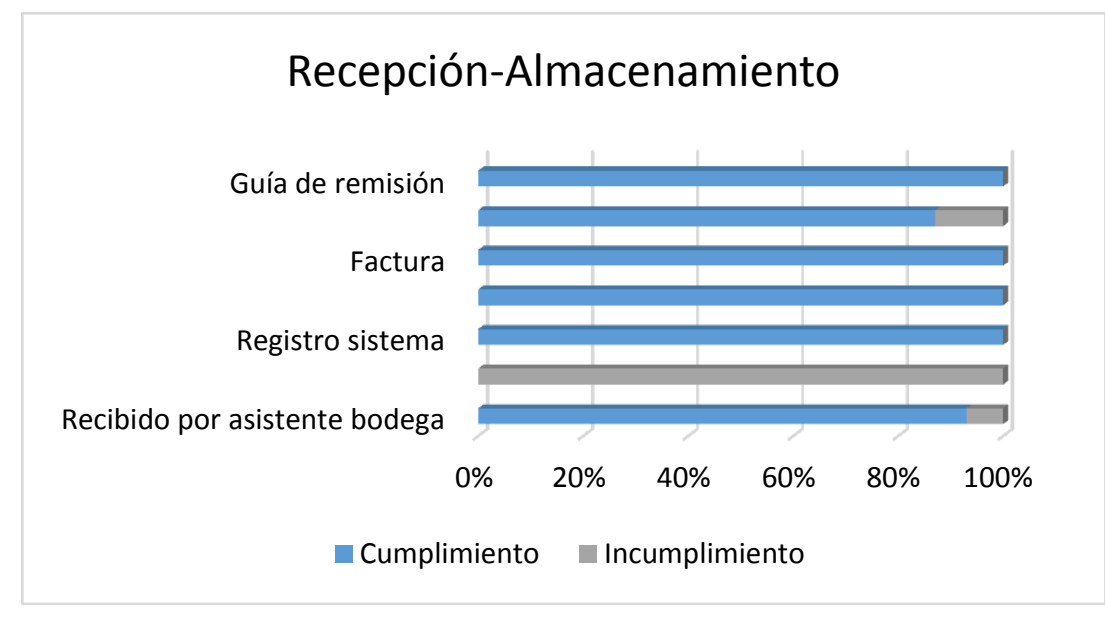

Figura 2. Cumplimiento de políticas en el proceso de recepción y almacenamiento Fuente: Investigación de campo.

\section{Nivel de cumplimiento de políticas en el proceso de recepción y almacenamiento}

El $100 \%$ de la mercadería que ingresa a la empresa es registrada por la secretaria en el sistema contable, pero al mismo tiempo existe un incumplimiento del $100 \%$ en el registro de los movimientos de la mercadería en la bodega del almacén. Según la NIC 2 los inventarios pueden ser valorados a través del método Promedio Ponderado o Método FIFO o PEPS, los cuales permiten conocer la cantidad de mercadería por artículo en bodega y el costo de los inventarios disponibles. Sin embargo, la empresa no cuenta con un documento físico que le permita registrar las entradas y salidas de la mercadería de bodega, con el objetivo de realizar 
reportes de inventario cuando sean necesarios. Lo que provoca desconocimiento del inventario físico que posee la empresa en las instalaciones o bodegas del local.

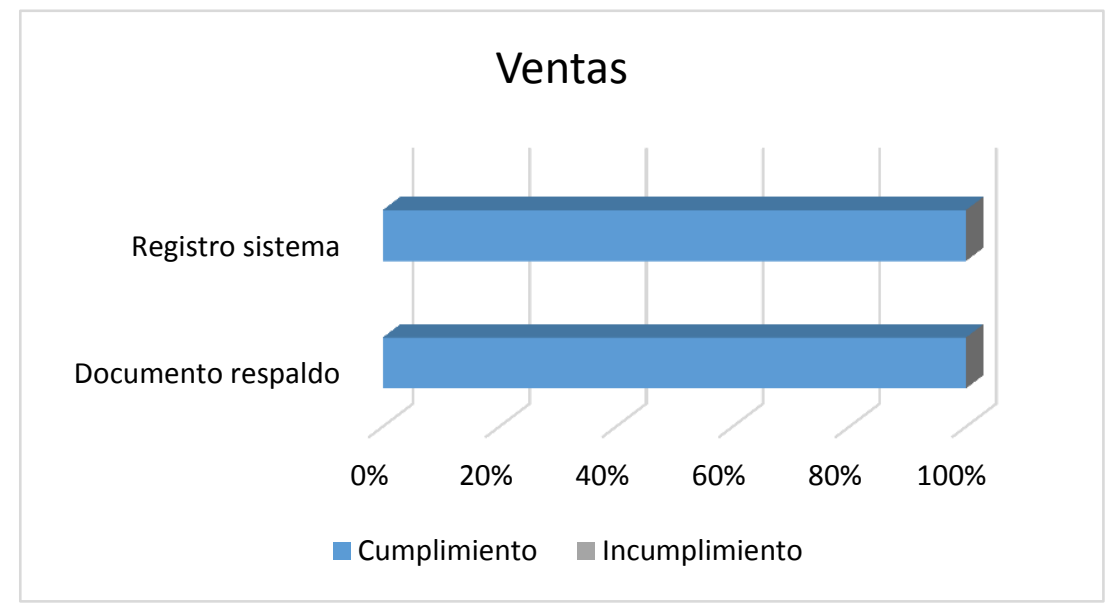

Figura 3. Cumplimiento de políticas en el proceso de ventas. Investigación de campo.

\section{Nivel de cumplimiento de políticas en el proceso de ventas}

Mediante una revisión física se pudo comprobar que la empresa sí cuenta con un documento respaldo de las ventas que realiza ya que posee archivado los tickets de venta que arroja el sistema con cada venta que la empresa realiza. De igual forma, se verificó que la empresa registra el $100 \%$ de las ventas que efectúa en el sistema contable que maneja.

Fase IV: Elaboración del informe acerca de los hallazgos encontrados en la evaluación del sistema de control interno

Como resultado de la evaluación del control interno de IMPORELLANA S.A, se determina que existe una serie de situaciones que afectan el control interno de la empresa, las cuales se especifican a continuación con su respectiva recomendación:

Ausencia de un Manual de funciones: La empresa no posee un manual de funciones que describa cada una de las actividades y tareas que debe realizar el personal que la conforma. Esto ocasiona que los empleados no realicen todas sus responsabilidades ya que no existen funciones o actividades definidas para cada puesto de trabajo. Además, conlleva a que los empleados no cumplan sus objetivos individuales ni los objetivos organizacionales. Por lo 
tanto, se recomienda elaborar un manual de funciones que ayude a la empresa a llevar un control sobre las tareas que debe desempeñar cada uno de los empleados, con el objetivo de organizar de una manera más eficaz el trabajo y evitar duplicidad de funciones.

Carencia de procedimientos para la gestión de inventarios: IMPORELLANA S.A no tiene documentos que detallen los procedimientos que los empleados deben seguir en cada uno de los procesos involucrados en la gestión de inventarios. Como consecuencia se genera una planificación deficiente de las compras que realiza la empresa, roturas de stock, deficiente registro de las entradas y salidas de mercadería de las bodegas de la empresa y por lo tanto un desconocimiento del inventario físico de la empresa. Se recomienda elaborar un manual de procedimientos, que describa todos y cada uno de los procesos que integran la secuencia de compra y venta de los productos que oferta la empresa, así como distribuir las responsabilidades que cada uno de los cargos de la empresa debe cumplir.

Deficiencia en la planificación de las compras: La empresa no organiza la adquisición de los artículos que comercializa, ya que se solicita el reabastecimiento de un producto cuando este se encuentra en niveles muy bajos de stock. Como consecuencia se producen rupturas de stock lo que genera consumidores insatisfechos por falta de mercadería para satisfacer sus necesidades de compra, y por ende, una disminución de las ventas. Se recomienda identificar artículos de alta y baja rotación, para conocer cuáles son los productos más demandados por los clientes. Además se debe establecer un nivel de mínimos y máximos de unidades para cada artículo que la empresa comercializa con el fin de no generar una rotura de stock así como un sobreabastecimiento de los productos que posee la empresa.

Carencia de documentos de respaldo en el proceso de compras: IMPORELLANA S.A no posee ningún documento que permita controlar y corroborar las características y cantidades de la mercadería que adquieren a un precio previamente convenido con sus proveedores. Esto genera incertidumbre en la eficiencia del pedido al momento de recibir la 
mercadería en las instalaciones de la empresa ya que los empleados solo revisan la nueva mercadería con la guía de remisión realizada por el proveedor. Se recomienda elaborar un formato de orden de compra que permita conocer las unidades, precios y descuentos de cada compra que realizan, así como la fecha de entrega y forma de pago de cada proveedor, esto con el objetivo de tener un respaldo físico al momento de recibir las mercaderías en las instalaciones de la empresa.

Ausencia de registros internos de la mercadería en el área de bodega: La empresa no posee ningún documento que permita registrar los movimientos, entradas y salidas, de la mercadería de las bodegas que posee la empresa. Esto genera un desconocimiento del inventario físico que posee IMPORELLANA S.A ya que solo se registran los movimientos en el sistema contable que utiliza la empresa. Se recomienda primero establecer un método de valoración de inventario, ya sea Promedio Ponderado o PEPS, de acuerdo al giro de negocio de la empresa. Elaborar un formato que permita registrar la cantidad, valor unitario y valor total de las compras y ventas que realiza la empresa, obteniendo de manera inmediata el saldo físico y valor respectivo de cada artículo.

\section{Conclusiones}

Mediante el levantamiento de información se pudo comprobar que Importadora Robert no tiene establecido de manera formal las funciones, políticas, procesos y documentos que el personal de la empresa debe cumplir y realizar para llevar a cabo una eficiente gestión de los inventarios que comercializa.

La evaluación del control interno se realizó con base en el cuestionario COSO ERM para detectar las deficiencias actuales en la gestión de inventario, la cual dio como resultado un nivel de riesgo alto. Por tanto, los controles aplicados por la empresa en el área estudiada son insuficientes, lo que da como consecuencia una incorrecta administración del inventario. 
La identificación de riesgos permitió conocer que el área crítica de la gestión de inventarios es de manera principal el proceso de compras y luego el proceso de recepciónalmacenamiento, ya que en ambos procesos existe un nivel alto de incumplimiento en las políticas de la empresa.

Se realizó el informe de auditoría con base a los hallazgos encontrados a partir de los cuales se elaboraron conclusiones y recomendaciones, para mejorar la administración de los inventarios y la toma de decisiones de la entidad.

\section{Referencias bibliográficas}

Belmonte, M. y González, N. (2013). Guía práctica para la realización de trabajos. España: Edit.um.

Deza, J. y Muñoz, S. (2012). Metodología de la investigación científica. Perú: Universidad a las peruanas.

El Telégrafo (26 de septiembre de 2012). La producción de línea blanca creció un $72 \%$ en cinco años. Recuperado de https://www.eltelegrafo.com.ec/noticias/2012/8/la-produccionde-linea-blanca-crecio-un-72-en-cinco-anos

Hernández, R., Fernández, C. \& Baptista, P. (2014). Metodología de la investigación. México: Mc Graw Hill.

Instituto Nacional de Estadística y Censo [INEC]. (2016). Encuesta Estructural Empresarial ENESEM 2016. Recuperado de http://www.ecuadorencifras.gob.ec/documentos/webinec/Estadisticas_Economicas/Encuesta_Estructural_Empresarial/2016/Tomo_I/2016_EN ESEM_Principales\%20Resultados.pdf

Obispo, D. y Gonzales, Y. (2015). Caracterización del control interno en la gestión de las empresas comerciales del Perú 2013. In Crescendo. Institucional; 6 (1), 64-73. Recuperado de https://dialnet.unirioja.es/servlet/articulo?codigo=5127579 
Paiva, F. (2013). Control interno y su incidencia en la rentabilidad de empresa cobros del norte S.A. Revista Científica de FAREM-Estelí, (7), 34-42. Recuperado de: https://www.lamjol.info/index.php/FAREM/article/view/2630

Quinde, C. y Ramos, T. (2018). Valuación y control del inventario y su efecto en la rentabilidad. Recuperado de https://www.eumed.net/rev/oel/2018/07/inventariorentabilidad.html

Vásquez, O. (2016). Visión integral del control interno. Contaduría Universidad de Antioquia, 1 (69), 139-154. Recuperado de: https://search.proquest.com/business/docview/1927854811/fulltextPDF/D13EB36712842 8APQ/2?accountid=13357

Vega, L., Lao, Y. y Nieves, A. (2017). Propuesta de un índice para evaluar la gestión del Control Interno. Contaduría y Administración, 62 (2), 683-698. Recuperado de http://www.sciencedirect.com/science/article/pii/S0186104217300049 